\title{
CORRIGENDUM
}

\section{Upper-limit power for self-guided propagation of intense lasers in underdense plasma - CORRIGENDUM}

Wei-Min Wang, Zheng-Ming Sheng, Yu-Tong Li, and Jie Zhang

doi:10.1017/hpl.2013.12. Published online by Cambridge University press 29 August 2013

The authors would like to apologize for an error found in the acknowledgement section on page 79 .

The acknowledgement should read:

This work is supported in part by National Basic Research Program of China (Grant No. 2013CBA01501) and National Natural Science Foundation of China (Grant Nos. 11105217, 11121504, 11129503, and 10925421). Numerical simulations have been partially carried out on 'Magic Cube' at Shanghai Supercomputer Center.

\section{Reference}

W.-M. Wang, Z.-M. Sheng, Y.-T. Li, and J. Zhang, Upper-limit power for self-guided propagation of intense lasers in underdense plasma. High Power Laser Science and Engineering 1, 74-79 (2013). 